\title{
Astrocyte origin of
} activity-dependent release of ATP and glutamate in hippocampal slices: real-time measurement utilizing microelectrode biosensors

\section{Correspondence}

Henry Sershen, Nathan Kline Institute, 140 Old Orangeburg Road, Orangeburg, NY 10962, USA. E-mail:

sershen@nki.rfmh.org

\section{Keywords}

glia; astrocytes; transmitter release; ATP; glutamate; adenosine; $\mathrm{K}^{+}$-depolarization; microelectrode biosensors

\section{Received}

15 May 2012

Revised

30 May 2012

Accepted

7 June 2012

\author{
Henry Sershen \\ Nathan Kline Institute, Orangeburg, NY, USA
}

It is well known that astrocytic and neuronal transmitter release processes are important for signalling, and that activity-dependent release of adenosine nucleotides and transmitters occurs after stimulation. Neurons and astrocytes can account for the source of ATP efflux. In this issue of the BJP, Heinrich et al. characterized $\mathrm{K}^{+}$depolarization-evoked release of ATP, adenosine and glutamate in hippocampal slices, utilizing microelectrode biosensors for simultaneous real-time recordings of multiple transmitter effluxes. They demonstrated efflux of ATP, adenosine and glutamate from hippocampus slices, in response to $\mathrm{K}^{+}$-depolarization, with distinct kinetics and mechanisms, suggesting a coordinated pattern of transmitter release. Surprisingly, it turned out that a considerable amount of the transmitter efflux measured under these conditions had a glial origin. For a long time, it was believed that the glial cell did not play a major role in neurotransmission, but the latter results somewhat change this view. The release of ATP and glutamate from glial cells under these conditions involved P2X7 receptors, and a source of adenosine accumulation independent of the metabolism of extracellular ATP was identified. This study also highlighted a novel use of multi-enzymatic microelectrode biosensors, which enabled a better characterization of transmitter release processes with higher temporal and spatial resolution than obtained previously. This technique was originally developed and used for the detection of purine release. In the present study, it was modified to identify the interplay between different transmitters, measured simultaneously in hippocampal slices.

\section{LINKED ARTICLE}

This article is a commentary on Heinrich et al., pp. 1003-1020 of this issue. To view this paper visit http://dx.doi.org/ 10.1111/j.1476-5381.2012.01932.x

It is accepted that signalling through the release of ATP is a predominant means of communication between glial and neuronal cells (Franke et al., 2012). There is considerable evidence showing that both the purine nucleoside adenosine acting on adenosine receptors and extracellular ATP acting on the P2-family of purinoceptors to release glutamate cooperate in the glia/neuron signalling pathway (Sperlagh et al., 2002; Anderson and Nedergaard, 2006; Marcoli et al., 2008; Sperlagh and Vizi, 2011). The mechanisms responsible for the control of this glial-neuronal cross-talk and its fine adjustment during neuronal activity, and what cells and receptor types, and from which vesicular pools these transmitters are released, and whether they act in concert or independently, have been major topics of interest. Understanding how the interactions of neurons and astrocytes regulate neurotransmitter release processes, which enable communication and control of synaptic cross-talk during neural transmission, and any changes that occur during pathological conditions, will further help in our elucidation of synaptic dysfunction in neurodegenerative diseases.

However, the current techniques for characterizing the endogenous source of transmitters and their sequence of 
release into the extracellular space have limitations. For example, with microdialysis measurements recovery is on average only $15-20 \%$ of the extracellular transmitters, and has a much delayed and prolonged temporal resolution that limits real-time calculations of efflux. Electrophysiological techniques can only indirectly detect transmitter release. In the study by Heinrich et al. (2012) these limitations were addressed by utilizing a recently developed enzymatic microbiosensor technique, originally used for the detection of purine release (Dale, 1998; Dale and Frengueli, 2012). This technique enabled real-time measurements of transmitter release that can further delineate processes of transmitter release with improved spatial and temporal reliability and accuracy. Utilizing these enzymatic biosensors may help to identify the 'trigger(s)' and pattern of transmitter(s) release occurring during synaptic activity. As the present paper demonstrated, the appearance, peak and decay of ATP, adenosine and glutamate responses were not identical with the same stimulation paradigm, suggesting a coordinated pattern of transmitter release. Surprisingly, it turned out that a considerable amount of the transmitter efflux measured under these conditions had a glial origin. One limitation of this technique was that the microelectrode biosensors were not able to measure the 'trigger' of glutamate efflux separately. The NMDA receptors that mediated ATP release were most likely to be on glial cells; however, the biosensor's resolution was limited and only AMPA-mediated glutamate release could be measured. The release of ATP and glutamate from glial cells under these conditions involved P2X7 receptors, and a source of adenosine accumulation that was independent of metabolism from extracellular ATP breakdown was also identified. The results indicated that adenosine was not only obtained from the metabolism of ATP, but also by a direct release of ATP.

There are still many processes involved in neuronal activity that are either unknown or have not been elucidated. Recently, both small vesicles and lysosomes have been shown to contribute to $\mathrm{Ca}^{2+}$-dependent vesicular exocytosis, and small vesicles support glutamate release from astrocytes (Liu et al., 2011). Lysosomes were shown to store ATP and release it when they fuse (Zhang et al., 2007). P2X receptors are expressed on glutamatergic excitatory postsynaptic synapses (Rubio and Soto, 2001), and can elicit both vesicular and non-vesicular transmitter release. In the present study, an additional complexity of efflux was suggested by the finding that glutamate, but not ATP release, was independent of $\left[\mathrm{Ca}^{2+}\right]_{0}$. This indicates that P2X7mediated glutamate release is non-vesicular, while P2X7mediated ATP efflux can be either vesicular or non-vesicular. Future studies are needed to understand whether different types of stimulation preferentially induce fusion from one or both compartments, and to determine whether exocytosis of small vesicles and lysosomes play specific roles in brain function in vivo.

Even more recently, microglia have also been added as potential upstream partners of astrocytes. When activated they release ATP, which triggers an enhancement of astrocytemediated modulation of transmitter release (ATP). Microglia release small amounts of ATP and astrocytes, in turn, amplify this ATP release (Pascual et al., 2012), suggesting an additional hierarchy in glial-neuronal cross-talk. These astrocytes, activated by microglia-released ATP, modulate neuronal activity at AMPA receptor-containing synapses by sequentially releasing glutamate that acts on neuronal metabotropic glutamate receptors.

It is also important to recognize that there are still many other unanswered questions with regard to the complex mechanisms involved in glial-neuronal cross-talk and synchronization. In addition to ATP, adenosine and glutamate, other factors and transmitters respond to synaptic stimulation and must be taken into account; for example, NO, trophic factors and cytokines. The physiological role and involvement of glucose oxidative metabolism in astrocytes for the supply of ATP or release of glutamate, and how it relates to energetics or to the control of signalling function, must be considered. The pharmacological profile of NMDA receptors expressed on astrocytes and neurons are substantially different, allowing selective modulation of astrocytic signalling (Palygin et al., 2011). In hippocampal slices proteinase-activated receptor- 2 activation induces a profound long-term depression of synaptic transmission that is dependent on NMDA receptor activation and is sensitive to disruption of astrocytic function, indicating that these receptors also modulate synaptic activity (Gan et al., 2011). Similar direct biosensor-based measurements in vivo have been used to show that the state of 'wakefulness' can affect mouse hippocampal astrocyte-derived adenosine (Schmitt et al., 2012). Wakefulness increases the level of extracellular adenosine and this is derived from astrocytes.

The ability of therapeutic agents to target specific synaptic responses can only be ascertained by using techniques that enable a resolution at the cellular and temporal level and are focused at understanding these complex cellular interactions. It is possible that microelectrode biosensor-based measurements can enable the clarification of these processes.

\section{Conflicts of interest}

The author has no conflicts to disclose at this time.

\section{References}

Anderson CM, Nedergaard M (2006). Emerging challenges of assigning P2X7 receptor function and immunoreactivity in neurons. Trends Neurosci 29: 257-262.

Dale N (1998). Delayed production of adenosine underlies temporal modulation of swimming in frog embryo. J Physiol 511 (Pt. 1): 265-272.

Dale N, Frenguelli BG (2012). Measurement of purine release with microelectrode biosensors. Purinergic Signal 8: 27-40.

Franke H, Verkhratsky A, Burnstock G, Illes P (2012). Pathophysiology of astroglial purinergic signalling. Purinergic Signal 8: 629-657.

Gan J, Greenwood SM, Cobb SR, Bushell TJ (2011). Indirect modulation of neuronal excitability and synaptic transmission in the hippocampus by activation of proteinase-activated receptor-2. Br J Pharmacol 163: 984-994. 
Heinrich A, Andó R, Túri G, Rózsa B, Sperlágh B (2012). K+ depolarization evokes ATP, adenosine and glutamate release from glia in rat hippocampus: a microelectrode biosensor study. Br J Pharmacol 167: 1003-1020.

Liu T, Sun L, Xiong Y, Shang S, Guo N, Teng S et al. (2011). Calcium triggers exocytosis from two types of organelles in a single astrocyte. J Neurosci 31: 10593-10601.

Marcoli M, Cervetto C, Paluzzi P, Guarnieri S, Alloisio S, Thellung S et al. (2008). P2X7 pre-synaptic receptors in adult rat cerebrocortical nerve terminals: a role in ATP-induced glutamate release.

J Neurochem 105: 2330-2342.

Palygin O, Lalo U, Pankratov Y (2011). Distinct pharmacological and functional properties of NMDA receptors in mouse cortical astrocytes. Br J Pharmacol 163: 1755-1766.

Pascual O, Ben AS, Rostaing P, Triller A, Bessis A (2012). Microglia activation triggers astrocyte-mediated modulation of excitatory neurotransmission. Proc Natl Acad Sci USA 109: E197-E205.
Rubio ME, Soto F (2001). Distinct localization of P2X receptors at excitatory postsynaptic specializations. J Neurosci 21: 641-653.

Schmitt LI, Sims RE, Dale N, Haydon PG (2012). Wakefulness affects synaptic and network activity by increasing extracellular astrocyte-derived adenosine. J Neurosci 32: 4417-4425.

Sperlagh B, Vizi ES (2011). The role of extracellular adenosine in chemical neurotransmission in the hippocampus and basal ganglia: pharmacological and clinical aspects. Curr Top Med Chem 11: $1034-1046$.

Sperlagh B, Kofalvi A, Deuchars J, Atkinson L, Milligan CJ, Buckley NJ et al. (2002). Involvement of P2X7 receptors in the regulation of neurotransmitter release in the rat hippocampus. J Neurochem 81: 1196-1211.

Zhang Z, Chen G, Zhou W, Song A, Xu T, Luo Q et al. (2007). Regulated ATP release from astrocytes through lysosome exocytosis. Nat Cell Biol 9: 945-953. 